\title{
Analysis of Spatial Pattern and Influencing Factors of Traditional Villages in Guangdong Province
}

\author{
Sun Xiliang \\ College of Urban and Environmental Sciences \\ Northwest University \\ Xi'an 710127, China \\ Liu Xiaoqiong \\ College of Urban and Environmental Sciences \\ Northwest University \\ Xi'an 710127, China \\ Wang Zhao \\ College of Urban and Environmental Sciences \\ Northwest University \\ Xi'an 710127, China
}

\author{
Wu Zezhou \\ College of Urban and Environmental Sciences \\ Northwest University \\ Xi'an 710127, China \\ Ma Teng \\ College of Urban and Environmental Sciences \\ Northwest University \\ Xi'an 710127, China \\ Rui Yang* \\ College of Urban and Environmental Sciences \\ Northwest University \\ Xi'an 710127, China \\ ruiyang@nwu.edu.cn
}

\begin{abstract}
The traditional village carries the important memory of the development of farming civilization in our country. To reasonable protect and utilize the traditional village, this paper takes Guangdong Province as an example, search for the differentiation and influence factors of 278 traditional villages, finally puts forward some suggestions to promote the overall protection of traditional villages. To estimate the differentiation, this paper uses the nearest neighbor index method, the kernel density analysis method and the hot spot analysis method. Then choose the terrain, hydrology and human factors as the starting point to test the influencing factors. The results visualized by ArcGis10. 2 shows that traditional villages tend to concentrated and have a greater correlation with terrain, hydrology and human factors.
\end{abstract}

Keywords-Traditional village; spatial pattern; Guangdong Province

\section{INTRODUCTION}

The traditional village is the village which is formed earlier, has rich traditional resources, and has certain historical, cultural, scientific, artistic, social and economic value[1]. Since 1950s, the new rural construction in china has made the traditional villages fade away gradually. Fortunately, in April 2012, the Ministry of housing and urban rural development, the Ministry of culture, the State Bureau of cultural relics, the Ministry of Finance jointly launched a survey of Chinese traditional villages, so that the protection and utilization of traditional villages have been gradually concerned.

The formation and continuation of traditional villages are related with village scale, layout and culture, with obvious spatial variation. At present, there are few researches on the

Sponsor: National Natural Science Foundation of China(No. 41601174), Social Science Foundation of Shaanxi Province, China (No. 2015D055), The Science Foundation of Northwest University(No. 14NW03)

*Corresponding author: E-mail: ruiyang@nwu.edu.cn spatial distribution of traditional villages in China, and most of the research findings focus on the provincial and municipal scale. Relatively speaking, the research on county scale is less[2-4]. The existing researches on traditional villages in Guangdong province are mostly focused on the form and architectural style of the villages[5-7], and lack of the study on the spatial distribution of traditional villages. Under the background of the comprehensive protection of traditional villages, the spatial interpretation of the traditional villages in Guangdong province is conducive to the protection of the relevant departments in the macro policy formulation, also is a great significance to the protection and utilization of the traditional village. This paper attempts to analyze the spatial distribution of traditional villages in Guangdong Province on the basis of the previous research results, and use GIS technology to study the influencing factors of the distribution of traditional villages. Finally, according to study results provide guidance and recommendations for the protection and utilization of traditional villages in Guangdong.

\section{DATA AND METHOD}

\section{A. Data sources and processing}

The traditional village data from the Ministry of housing and urban rural development, the Ministry of culture, the State Bureau of cultural relics, the Ministry of Finance. Since 2012, the published number of "Chinese traditional villages" in Guangdong Province is 126, then plus 186 traditional villages announced from Guangdong provincial government since 2014, and remove the 34 repeat data, finally the sample is 278 . Traditional village geographic coordinates by Google Earth software calibration. 90-m resolution DEM data download from geographic data cloud platform. Administrative divisions 
map originating from the geographic database. All the maps are based on ArcGis10. 2 and CrimeStat as a technical platform to visualize the geographical features of traditional villages.

\section{B. Research method}

1) The nearest neighbor index method. The distance between the relative position of the geographical distribution and the nearest neighbor is the important feature of the point element, and most commonly used the nearest neighbor index method to estimate the distribution of point elements. Specific methods refer to reference[8].

2) The kernel density analysis method. The kernel density estimation method is used to characterize the spatial influence area by attenuating bandwidth. Think of geographical events can occur in any position, but the probability of occurrence in different positions is not the same, the denser of point in an area, the higher the probability of the occurrence of geographical events. This method is usually used to measure the spatial equilibrium of point elements.

3) The hot spot analysis method. According to the position of the point, the hot spot analysis method generates the smallest outer ellipse, compares the number of points in the ellipse and the ellipse area to obtain the number of unit area, which higher than the average value is considered as hot spot area[9]. It is often used to detect the concentration distribution area of event points.

\section{THE CHARACTERISTICS OF GUANGDONG TRADITIONAL VILLAGE SPATIAL PATTERN}

\section{A. Spatial distribution type}

The nearest neighbor index can be calculated by using the spatial statistical tool of ArcGis10. 2, the result is as follows: Actual nearest neighbor distance is 0.094006 , theory nearest neighbor distance is 0. 155481, Point density is 0.60416 . therefore the ratio of the actual nearest neighbor mean and the theory nearest neighbor mean is less than 1 , and the $\mathrm{Z}$ score is 12. 611859. Preliminary judgment, the traditional villages of Guangdong Province, tends to agglomerate spatially distributed structure.

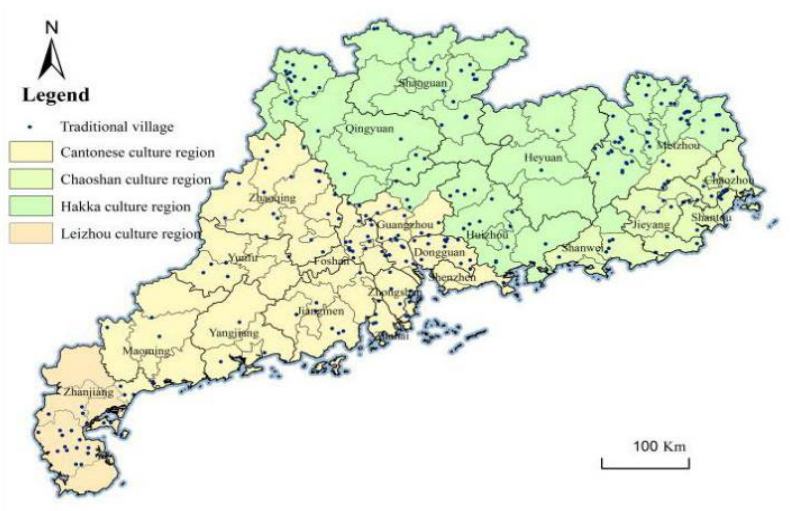

Fig. 1. The spatial distribution of traditional villages in Guangdong

\section{B. Spatial agglomeration area}

Spatial equilibrium can be measured by the distribution density. The kernel density analysis of 278 traditional villages is carried out by using the kernel density tool integrated with ArcGis10. 2 software. Upon examination, choosing the picture of kernel density distribution of $60 \mathrm{~km}$ bandwidth and the hotspot analysis, then stack it together. Finally find that the traditional villages in Guangdong have obvious characteristics of spatial agglomeration. The agglomeration areas are located in the eastern part of the Cantonese Cultural District, the eastern part of the Chaoshan Cultural District, the eastern part of the Hakka Cultural District and the northwest part, which is roughly coincident with the first hot spots. From the perspective of hotspot analysis, there are two second hot spots, which distributed in the eastern part of the Cantonese Cultural District, the middle part of the Hakka Cultural District, the northwest of Chaoshan Cultural District and the middle part of Leizhou Cultural District. In contrast, the area outside the hot spots due to the inconvenience of traffic, the insufficient number of villages and other reasons, the degree of concentration is not obvious.

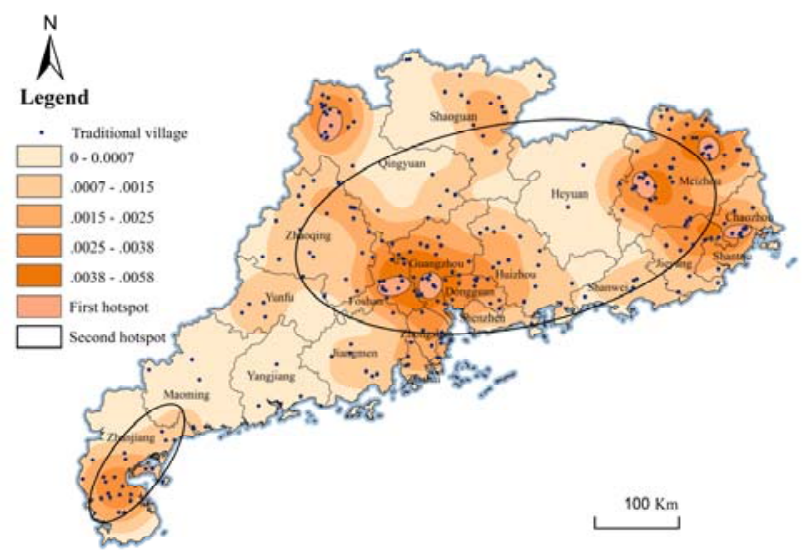

Fig. 2. The hot spot detection and kernel density distribution of traditional village in Guangdong

\section{INFLUENCING FACTORS OF THE CHARACTERISTICS OF Guangdong Traditional Village Spatial Pattern}

\section{A. Terrain factor}

1) Relief Amplitude. Using ArcGis10. 2 relief amplitude function to measure the topography of the surrounding villages. Among them, the relief amplitude is the average difference between the elevation of the highest and the lowest elevation in a specified area. It can be used to evaluate the characteristics of a regional terrain at the macro level. The results show that the location of the traditional village relief concentrated in $0 \sim 50 \mathrm{~m}$, that is, within the range of $900 \mathrm{~m} *$ $900 \mathrm{~m}$ around the geometric center of the traditional village, the number of villages with terrain elevation of $0 \sim 50 \mathrm{~m}$ is 194 , accounted for $69.78 \%$ of the total number. It reflects that the traditional villages in Guangdong province are distributed in relatively flat terrain area. There are $12.95 \%$ of the villages located in which terrain difference of more than 100 meters, 
most of these villages are surrounded by hills and mountains, which hinder the process of rapid urbanization, and form a relatively balanced self-organization environment.

2) Slope. The different orientations of the mountains represent the difference of sunshine duration and solar radiation intensity. Then using the slope direction analysis tool of ArcGis10. 2, and find that the selection of the slope of the traditional village has a variety of characteristics due to the complex terrain. Overall, it is obvious that villages tend to site in the positive, and to the southeast, south, southwest to the main, both accounting for $50.36 \%$ of the total number. If the slope is divided into sunny and shady slope, then the number of village in the sunny slope (90 to 270 degrees) is 181 , and shady slope( 0 to 90 degrees, 270 to 360 degrees)is 97 , the proportion of two is about 1.8 to 1 , which means that traditional villages have a clear positive tendency.

\section{B. Hydrologic factor}

Hydrological factors have an important influence on the formation and preservation of traditional villages. Using the $90 \mathrm{M}$ resolution DEM data of Guangdong province to carry out the hydrological analysis, and find that $37.41 \%$ of the traditional villages are less than $5 \mathrm{~km}$ away from the main river. When the distance is more than $15 \mathrm{~km}$, the number of villages substantially reduced, only $18.34 \%$ of the traditional villages away from the main river of $20 \mathrm{~km}$ away. In addition to some villages in the Leizhou Peninsula, most of the villages are close to the main and second channel, the production conditions and living environment can meet the basic needs of the people, which is conducive to the formation and protection of traditional villages.

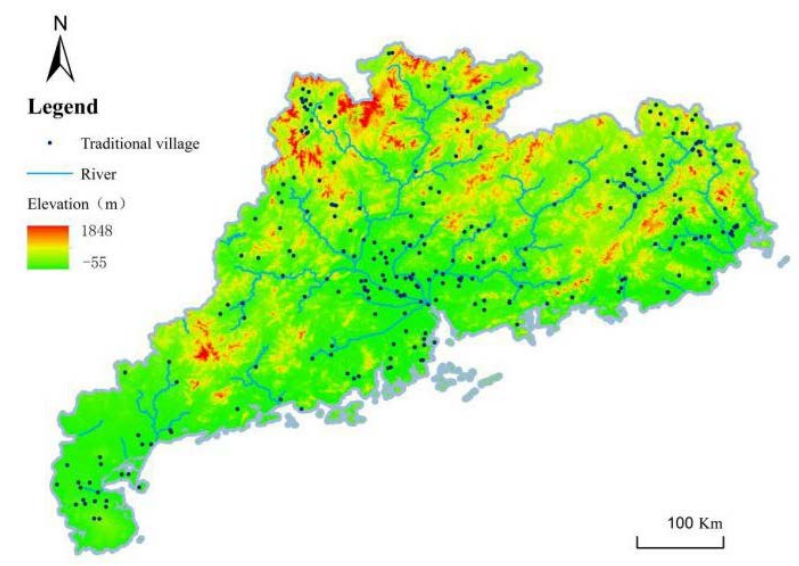

Fig. 3. The hydrological analysis of traditional villages in Guangdong

\section{Cultural factor}

Culture area as a region which has a continuous space range and a certain cultural landscape and it can be divided into cultural core area and the cultural diffusion area. When stack the traditional village location and the spatial distribution map, it is find that there are large differences in the number of traditional villages in different cultural areas, the proportion of Cantonese culture area and Hakka cultural area is $39.57 \%$, and the proportion of Leizhou Cultural District and Chaoshan Cultural District is respectively $13.67 \%$ and $7.19 \%$. According to the number of traditional villages within the cultural area, the core area of Cantonese, Hakka, Chaoshan and Leizhou accounted for $65.45 \%, 58.18 \%, 63.16 \%$ and $100 \%$, only in the Hakka culture area, there is a situation that the traditional villages gather in the cultural diffusion area, and in contrast, the traditional villages in other cultural areas are generally concentrated in the cultural core area. It shows that the spatial distribution of traditional villages is affected by the origin of culture to some extent, and tends to be concentrated in the core area of culture.

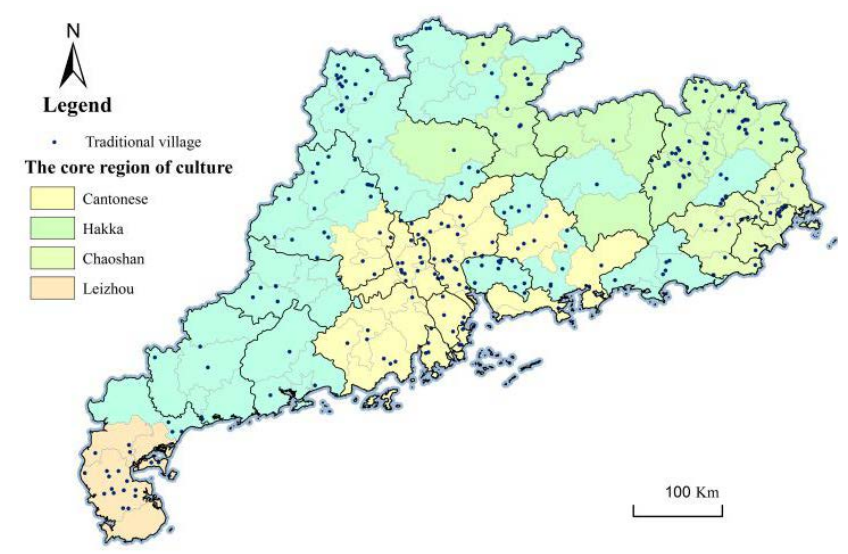

Fig. 4. The spatial distribution of core cultural areas in Guangdong

\section{CONCLUSION AND PROSPECT}

Restricted by nature, society, culture and many other factors, the spatial differentiation of traditional village is imbalanced. Both number and density of traditional villages in Guangdong Province is large, space agglomeration characteristic is obvious which shows dispersion in large scale and concentration in small scale. For spatial pattern, the traditional village of Guangdong Province tends to choose the location of its cultural transmission core area. On the choice of terrain, it tend to located in the area which has small terrain height difference, relatively continuous landforms and better traffic conditions. Most of the traditional village sites in the clear positive tendency and it tends to sit in the relatively flat slopes of upper river or the impact plains of the sea downstream.

Most of the traditional villages in Guangdong Province which close to each other and have similar economic and cultural ties are gathered in the vicinity of several centers to form a cultural circle. Thus, for the protection of these interrelated and mutual traditional village. First of all, government should not blindly according to the precedent planning to formulate traditional village protection plan. Decision making should be based on the differentiation among traditional villages to determine the different traditional cultural areas of the village and develop traditional village protection plan with target, according to the natural and social factors during its formation, development and continuation. Secondly, to help grassroots officials to work under law, government should improve the legal system of traditional 
villages and develop traditional village protection law. Current cultural-relics protection law only protects the cultural relics and historical cultural villages recognized which lacks the concern about the undelegated traditional villages.

This paper only takes 278 traditional villages in national and provincial levels of Guangdong as example. Only analyze its topography, spatial distribution but not take culture, internal structure of the village into consideration. The next step is to study the spatial differentiation of traditional villages and the unity of their internal cultural attributes.

\section{REFERENCES}

[1] TONG Y Q. Research on the spatial differentiation of Chinese traditional village based on GIS. Human Geography, 2014, (04):44-51. (In Chinese)

[2] LIU D J, HU J, CHEN J Z, XV X T. The study of spatial distribution pattern of traditional villages in China. China Population Resources and Environment, 2014, (04):157-162. (In Chinese)
[3] TONG Y Q, LONG H L. The Spatial Differentiation Factors of Ethnic Traditional Villages in Guizhou. Economic Geography, 2015, (03):133137. (In Chinese)

[4] LI B H, YIN S, LIU P L, DOU Y D. Spatial distribution of traditional villages and the influencing factors in Hunan Province. Economic Geography, 2015, (02):189-194. (In Chinese)

[5] ZHU X M. Research on the morphonlogy of traditional village and architectrural features in northern Guangdong. South China University of Technology, 2013. (In Chinese)

[6] LI Z B. The comparative study on the traditional village form in the Pearl River Delta and the southeast of Guangxi under the cultural background of Guangfu. South China University of Technology, 2014. (In Chinese)

[7] HUANG B C. The comparative study on the traditional dwelling houses of Chaoshan area and Leizhou area in Guangdong. South China University of Technology, 2016. (In Chinese)

[8] TIAN G J, SHA M Q. The spatial pattern of Guangzhou metropolitan area industry based on point data and GIS. Progress in Geography, 2010, (04):387-395. (In Chinese)

[9] WANG Z H. Temporal-spatial hot spot analysis on crime cases based on scan statistics methodologies in Shanghai. East China Normal University, 2013. (In Chinese) 